\title{
The influence of standing waves on synchronization and self-heating of Josephson junctions in resonant systems
}

\author{
Alexander Grib ${ }^{1,2}$ and Paul Seidel ${ }^{1}$ \\ ${ }^{1}$ Institut für Festkörperphysik, Friedrich-Schiller-Universität Jena, Jena D-07743, Germany \\ E-mail: paul.seidel@uni-jena.de \\ ${ }^{2}$ Physics Department, V.N. Karazin Kharkiv National University, 4 Svobody Sq., Kharkiv 61022, Ukraine
}

Received October 17, 2011

\begin{abstract}
We investigated numerically synchronization of Josephson junctions inside the transmission line. We have found that due to the resonance behavior of the system there appear the self-induced resonance steps, strong synchronization of junctions on these steps and the inhomogeneous distribution of the Joule heat extraction (the selfheating) along the line which can lead to the formation of "hot spots" in the line. The developed model can be applied to explain recent experiments in which these effects were obtained.
\end{abstract}

PACS: $05.45 . X t \quad$ Synchronization; coupled oscillators;

74.50.+r Tunneling phenomena; Josephson effects;

85.25.Cp Josephson devices.

Keywords: Josephson junctions, synchronization, high-temperature superconductors.

\section{Introduction}

Since the experimental proof of the non-stationary Josephson effect [1] the problems connected to the use of Josephson junctions as tunable sub-mm oscillators with a high precision of frequency become important because of the application of the sub-mm wave band sources in different fields of science. The main problem of Josephson junction oscillators is the phase locking of many junctions to get appreciable large output power. An easy way to solve this problem is to use a resonator to perform a phase locking feedback. Recently, immense progress in experiments on the detection of synchronized radiation from intrinsic Josephson junction arrays in high temperature superconductors (HTSC) was achieved [2-4]. It was supposed [2] that the array of intrinsic junctions itself can be a geometrical resonator. To explain the effect, the hypothesis of the nonuniform distribution of critical currents of intrinsic junctions was proposed [2] as well as the hypothesis about the existence of the plasma resonance in the system [5]. Experiments showed also that the temperature along the measured samples of intrinsic Josephson junctions can be strongly inhomogeneous [6] and the so-called "hot spots" (i.e., places in the sample where the temperature can even reach the critical temperature of the superconductor) can be formed $[7,8]$.

The hypothesis about the resonance properties of the array of intrinsic Josephson junctions assumes the formation of standing waves of the electromagnetic field inside this array. Earlier we developed models of synchronization of the arrays of junctions shunted by the resonant transmission line [9] and the resonator [10,11] and found that the current resonance is responsible for synchronization in both cases. In the present paper we consider the formation of a standing wave in the resonant transmission line with open ends and the influence of it on synchronization of junctions embedded in the line. We suppose that this structure can model the arrays of intrinsic junctions which were investigated experimentally. In our model instead of continues layers of intrinsic junctions there is a set of "elementary junctions" which form a two-dimensional array inside the transmission line (Fig. 1). We believe that this model is more adequate to the experiments in which the typical dimensions of the mesastructure is $300 \times 50 \mu \mathrm{m}$ and the structure can contain defects like dislocations and grain boundaries, whereas the distance between intrinsic Josephson junctions is only about $1.5 \mathrm{~nm}$. For the modeling we chose the simplest case of only two layers of insulator divided to the "elementary junctions". The pair of these "elementary junctions" belonging to different layers forms a stack. It is known [12] that the distance of electromagnetic interaction of junctions in a stack is infinitive, i.e., each of the junctions in the stack interacts with any other junction and this interaction does not decay with the number of the junction in the stack (i.e., with the "distance" between junctions). Therefore, main particularities of the electro- 
magnetic interaction between junctions can be discussed in the simple system of only two junctions in a stack. In the present paper we do not consider all features of the developed complicated model but concentrate on the main three particularities which were obtained experimentally: (i) that the measured system has the self-induced resonant steps in its $\mathrm{I}-\mathrm{V}$ characteristic, (ii) that the coherent radiation is registered at these resonant steps and (iii) that there is the strongly inhomogeneous distribution of the temperature along the radiating system that leads to the formation of the "hot spots". We obtain all these three features in our model and show that all of them have the same origin that is the resonant behavior of the system.

\section{The model}

The example of the high-frequency scheme of the considered system is presented in Fig. 1. The transmission line is divided on $N$ cells (in Fig. $1 N=6$, but this number can be varied). The length of the cell with the index $j$ is $\xi_{0}$. The value of $\xi_{0}$ coincides with the linear dimension of the "elementary junction" or the "grain". The geometry of the problem is as follows. The $x$-axis is directed along the transmission line (see Fig. 1). The first cell at the left edge of the transmission line has the index $j=1$. We call the value of $\xi_{0}(j-1 / 2)$ as the "distance" along the transmission line and will calculate "spatial" distributions of currents and voltages along this direction. Centers of "grains" coincide with positions of high-frequency current lines. The $z$-axis is directed along these high-frequency current lines and the $y$-axis is perpendicular to the plane of Fig. 1 . The size of the transmission line along the $y$-direction is not limited. The size along the $z$-direction is the sum of thicknesses of two superconducting layers and two layers of insulator.

Each of the cells of the transmission line in Fig. 1 has the capacitance $C$, the inductance $L$ and the resistance $R$. The two-dimensional array of junctions is notified by indices $i=1,2$ and $l=1, \ldots, N-1$, where the index $i$ is for the upper $(i=1)$ or the lower $(i=2)$ junction in the highfrequency electrical line which divides two neighbor cells and the index $l$ is for the number of this electrical line in the system. These two junctions in the high-frequency electrical line we call as a stack of junctions. Each of the

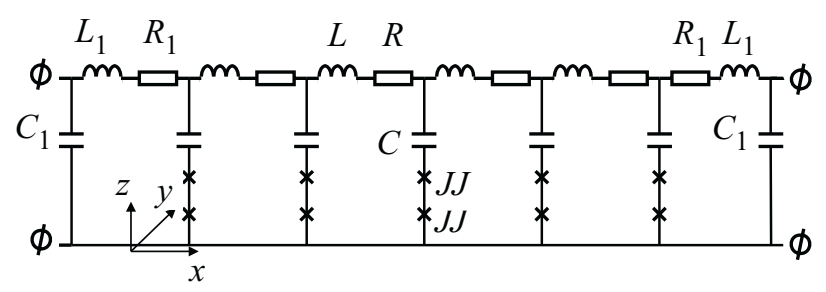

Fig. 1. The high-frequency scheme of the system which contains 6 cells and 5 stacks of junctions. The system considered in the paper contains 60 cells and 58 stacks. The $x$-position of the origin of coordinates is in the middle of the bottom line of the first cell. stacks has the source of the direct current. All sources for all stacks have the same value $I_{b}$. The direction of the current in the stack is from the lower junction to the upper junction. The set of junctions with the indices $i=1$ represents the upper layer of insulator divided on "grains" and the set of junctions with the indices $i=2$ represents the lower layer of insulator divided on "grains", correspondingly. For the simplicity we assume that all "grains" in both layers have the same size. Boundary conditions of the problem include the reflection of electromagnetic waves from ends of the line. These conditions were modeled by means of capacitances $C_{1}$, inductances $L_{1}$ and resistances $R_{1}$ at ends of the line.

Let us denote $\varphi_{i, l}$ as the phase difference of the order parameter across the $i$ th junction in the stack belonging to the high-frequency current line with the number $l$. Then the system of differential equations describing phase dynamics in the range of the model of the capacitively and resistively shunted junction [12] is as follows:

$$
\begin{gathered}
\frac{\Phi_{0} C_{0 i, l}}{2 \pi} \ddot{\varphi}_{i, l}+\frac{\Phi_{0}}{2 \pi R_{i, l}} \dot{\varphi}_{i, l}+I_{c i, l} \sin \varphi_{i, l}=I_{b}-\dot{q}_{j}+\dot{q}_{j+1} \\
l=1, \ldots, N-1, \quad j=l \\
L \ddot{q}_{j}+R \dot{q}_{j}+\frac{2 q_{j}}{C}=\frac{\Phi_{0}}{2 \pi}\left(\sum_{i=1}^{2} \dot{\varphi}_{i, l-1}-\sum_{i=1}^{2} \dot{\varphi}_{i, l}\right) \\
j=2, \ldots, N-1, \quad l=j, \\
L_{1} \ddot{q}_{1}+R_{1} \dot{q}_{1}+\frac{q_{1}}{C_{1}}=-\frac{\Phi_{0}}{2 \pi} \sum_{i=1}^{2} \dot{\varphi}_{i, 1}, \quad j=l=1 \\
L_{1} \ddot{q}_{N}+R_{1} \dot{q}_{N}+\frac{q_{N}}{C_{1}}=\frac{\Phi_{0}}{2 \pi} \sum_{i=1}^{2} \dot{\varphi}_{i, N-1}, j=N, l=N-1
\end{gathered}
$$

where $\dot{\varphi}_{i, l}$ and $\ddot{\varphi}_{i, l}$ are the first and the second time derivative of the phase difference, $q_{j}$ is the charge which passes the inductance of the $j$ th cell, $\Phi_{0}$ is the quantum of magnetic flux, $C_{0 i, l}$ and $I_{c i, l}$ are the capacitance and the critical current of the junction, correspondingly, $R_{i, l}$ is the resistance of the junction. For simplicity we keep all critical voltages across junctions $V_{c i, l}=I_{c i, l} R_{i, l}=V_{c}$ to be the same for all junctions.

\section{Results and discussion}

For calculations typical values of parameters for critical currents and critical voltages of intrinsic Josephson junctions [1] $I_{c}=15 \mathrm{~mA}, V_{c}=2 \mathrm{mV}$ were chosen. To obtain phase locking we consider the simplest artificial case of small spread of critical currents $\delta=0.02$ which is the same in each stack, so the upper layer of junctions has critical currents $I_{c}(1-\delta)$ and the lower layer of junctions has critical currents $I_{c}(1+\delta)$. Values of $L=1.62 \mathrm{fH}$ and $C=0.12 \mathrm{pF}$ were obtained to satisfy the value of the velocity of light ob- 
tained for the high-temperature superconductor [1] under the condition of the size of the junction $\xi_{0}=1 \mu \mathrm{m}$. For the boundary conditions we chose $L_{1}=L, C_{1}=C$ and $R_{1}=0$ (the transmission line with the open ends). We also introduced small Joule losses in the line $\left(R=10^{-4} \mathrm{Ohm}\right)$. To demonstrate the main results of the model in the present paper we use small values of the McCumber parameters of junctions $\beta_{C 0}=\left(2 \pi I_{c} R_{j}{ }^{2} C_{0}\right) / \Phi_{0}=1$. Though these values are smaller than those for high-temperature superconductors $\left(\beta_{C 0} \approx 10\right)$, we checked that main results of the model are saved for them too.

We investigated the system which contains 58 stacks. In Fig. 2 we present the I-V characteristics for the first stack from the left side of the system. The I-V characteristic contains resonant steps. Note that these steps are self-induced, i.e., they appear without the external irradiation but only as the result of the electromagnetic interaction of junctions inside the transmission line. It was shown [9] that the resonances in the system of Josephson junctions loaded by the transmission line appear when the averaged over time voltage across the junction becomes equal to the value $\langle V\rangle_{r n}=n V_{c} /(2 \chi)$, where $n$ is integer, $\chi$ is the length of the system in units of wavelengths of the ac current in the line and the sign $\langle\ldots\rangle$ means averaging over large time. Standing waves in the line appear at these resonant voltages. For the given parameters of the model the resonance voltage is equal to $\langle V\rangle_{r 1}=0.63 V_{c}$. The centers of steps in Fig. 2 match values of $\langle V\rangle_{r n}=n \cdot 0.63 V_{c}$ exactly. It is necessary to note that $\mathrm{I}-\mathrm{V}$ characteristics of stacks along the transmission line can be quite different from the I-V characteristic shown in Fig. 2 because the resonant conditions for them are

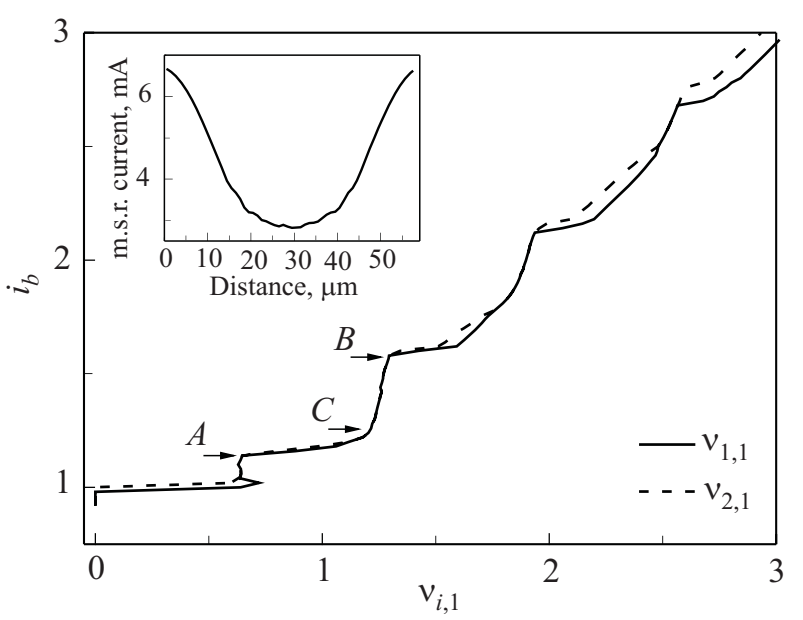

Fig. 2. I-V characteristics of individual junctions for the first stack from the left side of the transmission line plotted in normalized units: $\left\langle v_{i, 1}\right\rangle \equiv\left\langle V_{i, 1}\right\rangle / V$ and $i_{b}=I_{b} / I_{c}$. The plot of $\left\langle v_{i, 1}\right\rangle=f\left(i_{b}\right)$ is shown only for the increasing bias current. The I-V curve for the junction with smaller critical current is shown by the solid line. Points of the $\mathrm{I}-\mathrm{V}$ characteristic marked by arrows $A, B, C$ are discussed in the paper. Inset: the spatial distribution of the mean square root (m.s.r.) value of the ac current flowing through stacks at $i_{b}=1.14$ (the point $A$ in the $\mathrm{I}-\mathrm{V}$ characteristic). not satisfied. For example, for junctions in the centre of the line there exist only even numbers of the steps.

Junctions radiate coherently at the resonant steps. It is seen from Fig. 2 that they have the equal averaged voltages near the upper edges of steps that is characteristic for phase locked states. It is known [12] that in-phase locking is determined by the negative value of the imaginary part of the connection coefficient $Y_{i, l}$ of the electromagnetic interaction between junctions. For two junctions loaded by the transmission line with the open end the connection coefficient has tangential particularities at the resonant steps due to the current resonance [9], so the strongest phase locking is expected at upper edges of the resonant steps where $\operatorname{Im}\left(Y_{i, l}\right)<0$ and the absolute values of $\operatorname{Im}\left(Y_{i, l}\right)$ are maximal. The behavior of $\mathrm{I}-\mathrm{V}$ characteristics in Fig. 2 is in full agreement with this consideration. We checked also the presence of phase locking numerically considering the time dynamic of phase differences across junctions and proved that 14 stacks at the left end and 14 stacks at the right end of the transmission line radiate coherently. In inset of Fig. 2 we show the spatial distribution of the mean square root value of the ac current flowing through stacks. It is seen that the largest values of this current are at ends of the line. This current synchronizes junctions at ends of the line.

Distributions of voltages in stacks along the transmission line are shown in Fig. 3 for different values of the bias current which increases with the step $0.02 I_{c}$. There is one large irregularity of voltages in the center of the line in the vicinity of the voltage corresponding to the main resonant mode, two irregularities in the vicinity of the second resonant mode etc. These irregularities indicate the standing waves which appear at different dc currents.

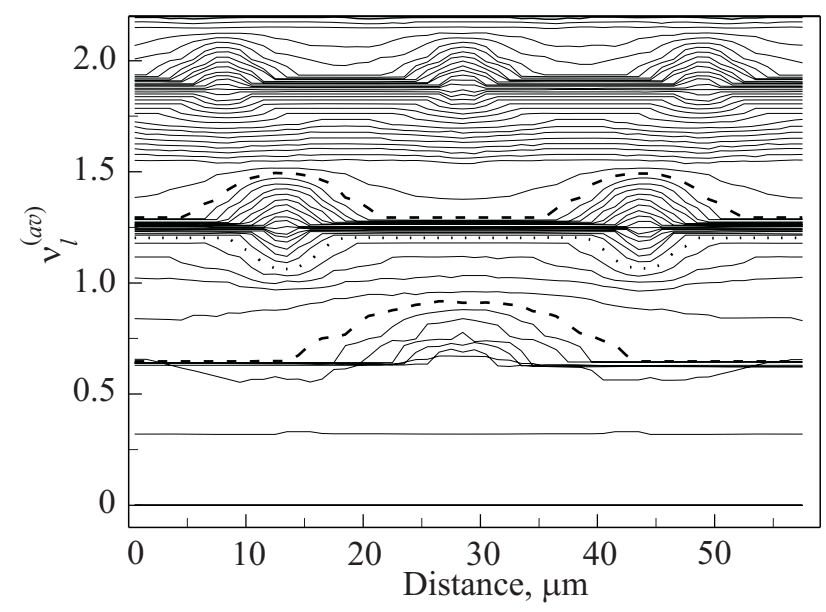

Fig. 3. The distributions of averaged voltages $v_{l}^{(a v)}=\left[\left(\left\langle V_{1, l}\right\rangle+\left\langle V_{2, l}\right\rangle\right) / V_{c}\right] / 2$ along the transmission line at different values of the bias current which increase with the step $0.02 I_{c}$. Distributions which correspond to currents $1.14 I_{c}$ and $1.58 I_{c}$ (points $A$ and $B$ in Fig. 2) are marked by the bold dashed line. The distribution which corresponds to the current $1.24 I_{c}$ (the point $C$ in Fig. 2) is marked by the bold dotted line. 
In the following consideration we discuss values of the averaged over time instant power in the stack $P_{l}=$ $=\left\langle\frac{\Phi_{0}}{2 \pi} \sum_{i=1}^{2} \dot{\varphi}_{i, l}\left(I_{b}-\dot{q}_{l}+\dot{q}_{l+1}\right)\right\rangle$ with $j=l$. This value is responsible for the Joule heat extracted from the given stack $l$. We also introduce the value of $S_{l}=\left\langle\left(\sum_{i=1}^{2}\left(\frac{\Phi_{0}}{2 \pi} \dot{\varphi}_{i, l}-\left\langle V_{i, l}\right\rangle\right)\right)^{2}\right\rangle$ that is the square of the ac voltage over the $l$ th stack. The value of $S_{l}$ is proportional to ac power of coherent radiation of junctions in the $l$ th stack $[10,12]$. If junctions in the stack radiate coherently with the zero phase shift, the value of $S_{l}$ increases up to $4 S_{i, l}$, where $S_{i, l}=\left\langle\left(\frac{\Phi_{0}}{2 \pi} \dot{\varphi}_{i, l}-\left\langle V_{i, l}\right\rangle\right)^{2}\right\rangle$ is the value that is proportional to ac power of the individual junction in the stack; if junctions radiate with the phase shift $\frac{\pi}{2}$ then $S_{l}=0$ and if the phase shift is random then $S_{l}=2 S_{i, l}$.

In Figs. 4, $a$ and 5, $a$ we plotted distributions of $P_{l}$ along the transmission line for the bias currents corresponding to the upper edges of steps (these points in the I-V characteristic are shown in Fig. 2 by arrows $A$ and $B$ and the corresponding distributions of voltages are shown in Fig. 3 by bold dashed lines). The distributions of $S_{l}$ for the same bias currents are plotted in Figs. $4, b$ and $5, b$. It is seen from Figs. 4, 5 that in the spatial places of the line where $P_{l}$ has a maximum, the value of $S_{l}$ decreases, and the maxima of $S_{l}$ appear in spatial places where values of $P_{l}$ are minimal.

The described effect is the main result of the present paper. Its physical meaning is as follows. The emitted power of synchronized radiation of junctions in the system
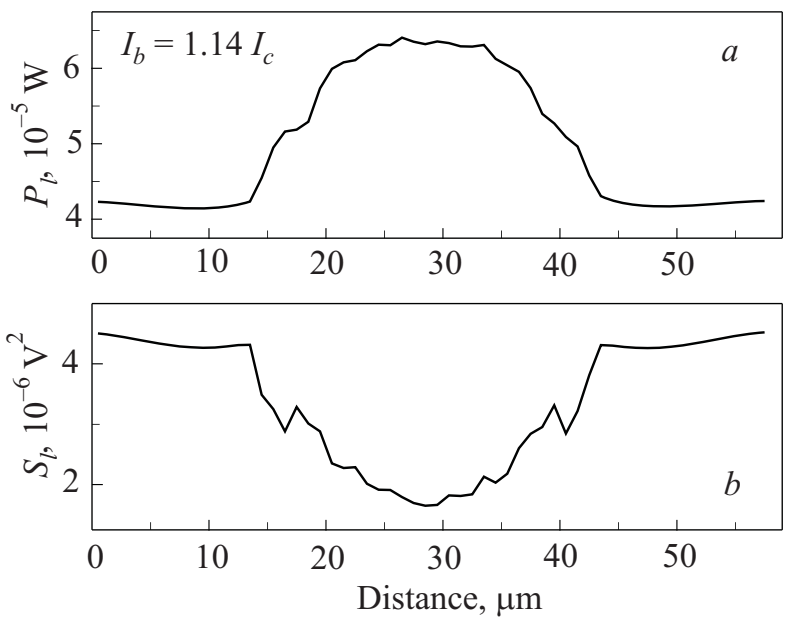

Fig. 4. Distributions of $P_{l}(a)$ and $S_{l}(b)$ along the transmission line for the bias current $I_{b}=1.14 I_{c}$ corresponding to the point $A$ in Fig. 2.
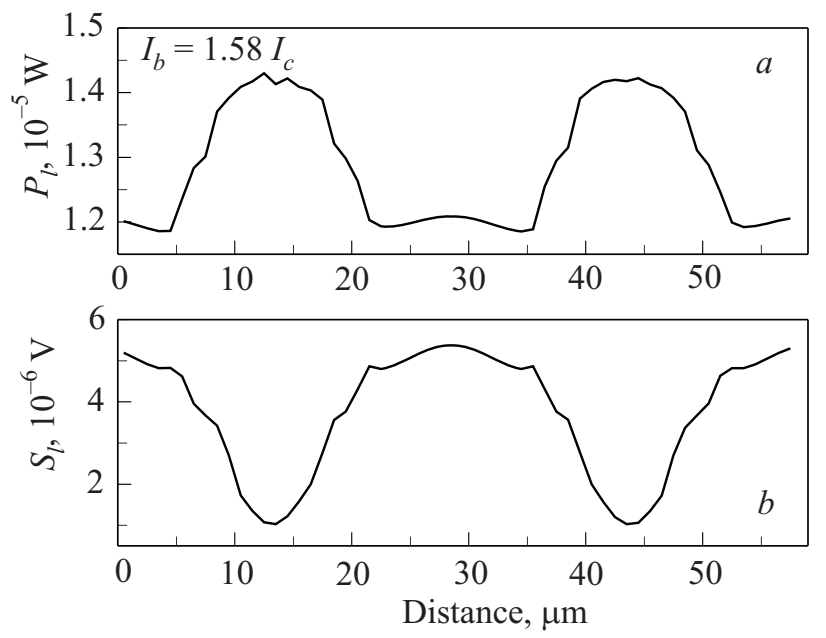

Fig. 5. Distributions of $P_{l}(a)$ and $S_{l}(b)$ along the transmission line for the bias current $I_{b}=1.58 I_{c}$ corresponding to the point $B$ in Fig. 2.

is maximal at the self-induced resonant steps (more precisely, at upper edges of steps). At the same time, the standing wave is formed at the resonant steps. Note that in this standing wave there is the increase of averaged on time voltages in the center of the line. Junctions cannot radiate coherently in the places where the standing wave exists because the averaged voltages over junctions have different values along the standing wave. Therefore, the parts of the line where junctions radiate coherently and the parts where the standing wave appears should be in spatially different places of the line.

It is seen from Fig. 4, $a$ that due to the standing wave the value of $P_{l}$ can increase as much as 1.5 times in some spatial places of the transmission line. This means that the Joule heat extraction is larger in these places, so the temperature in these places increases. In the model [13] the value of $P_{l}$ is proportional to the difference $\Delta T_{l}$ between the temperature of the overheating of the stack and the temperature of the cryostat: $\Delta T_{l}=\gamma_{l} P_{l}$, where the coefficient of proportionality $\gamma_{l}$ can be roughly estimated from the Fourier law of the stationary heat flux: $\gamma_{l}=\Delta Z_{l} / \lambda A$, where $\Delta Z_{l}$ is the characteristic distance along the $z$-direction at which the temperature changes, $\lambda$ is the thermal conductivity of the high-temperature superconductor and $A$ is the cross-section of the junction. The conditions of the experiment [2] claim that the main heat sinks are the free surface of the mesa and the volume of the high-temperature superconductor single crystal. The influence of the surface on the increase of the temperature cannot be calculated in the ranges of the Fourier law, therefore we considered only heat flux into the volume of the superconductor and therefore our estimate of the temperature of overheating is quite rough. Supposing $\Delta Z_{l}=1 \cdot 10^{-7} \mathrm{~m}$ for a stack of two junctions placed at the distance $0.1 \mu \mathrm{m}$ from the surface and substituting parameters of the model $A=10^{-12} \mathrm{~m}^{2}$ and $\lambda=8 \mathrm{~W} /(\mathrm{m} \cdot \mathrm{K})[14]$ into the expression 
for $\gamma_{l}$, we obtain from Fig. $4, a \Delta T_{l=30} \approx 0.79 \mathrm{~K}$ near the center of the transmission line and $\Delta T_{l=10} \approx 0.52 \mathrm{~K}$ near the edge of the line. Note that in our calculations of $\Delta T_{l}$ heat fluxes from junctions are added to each other. In the system of several hundred junctions in the stack the selfheating can cause the "hot spot" in the center of the sample for the first harmonic, see Fig. 4, $a$ or two "hot spots" for the second harmonic (see Fig. 5,a), whereas the surrounding junctions will radiate coherently. The detail calculations can be made with the use of the proposed model. Note that for the description of the experiments $[2-4,6-8]$ with the given width of the sample it is necessary to calculate distributions of voltages and currents also along the $y$-axis.

It is seen from Fig. 3 that in the vicinities of the second and higher harmonics of the resonance there is also the decrease of the averaged over time voltages. In Fig. 6 we plotted distributions of values of $P_{l}$ and $S_{l}$ for one of the bias currents which corresponds to such a decrease (it is marked by the arrow $C$ on the $\mathrm{I}-\mathrm{V}$ characteristic in Fig. 2 and the corresponding distribution of averaged over time voltages is shown by the bold dotted line in Fig. 3). The values of $P_{l}$ decreases in the places of the line where the averaged on time voltage decreases (Fig. 6,a), so there should be the decrease of the temperature of overheating. Values of $S_{l}$ are much smaller than those obtained at the upper edge of this step, so the coherent radiation in this case is small.

\section{Conclusions}

In the present paper we obtained straightforward the standing wave in the transmission line with Josephson junctions and analyzed synchronization of junctions under the influence of the standing wave. In our model we obtained self-induced resonant steps on I-V characteristics of junctions, explained their appearance and found resonant voltages. We showed that strong synchronization of junctions with different critical currents appears at these resonant steps due to the current resonance. We showed that


Fig. 6. Distributions of $P_{l}(a)$ and $S_{l}(b)$ along the transmission line for the bias current $I_{b}=1.24 I_{c}$ corresponding to the point $C$ in Fig. 2. only junctions which are close to ends of the line are synchronized at resonant steps. The averaged on time voltage of junctions in the center of the line increases and forms the standing wave. Due to strong extraction of Joule heat (the self-heating) the temperature of junctions in the center of the line can exceed the temperature at ends as much as 1.5 times that can lead to the formation of the "hot spot" in the center of the line, whereas at the same time junctions at ends of the line radiate coherently. Thus, we showed that three particularities noted in experiments $[2-4,6-8]$, namely the self-induced resonant steps on I-V characteristics, strong coherent emission at these steps and the appearance of "hot spots" have the same origin that is the resonance behavior of the system at certain bias currents.

1. I.K. Yanson, V.M. Svistunov, and I.M. Dmitrenko, Sov. Phys. JETP 21, 650 (1965).

2. L. Ozyuzer, A.E. Koshelev, C. Kurter, N. Gopalsami, Q. Li, M. Tachiki, K. Kadowaki, T. Yamamoto, H. Minami, H. Yamaguchi, T. Tachiki, K.E. Gray, W.-K. Kwok, and U. Welp, Science 318, 1291 (2007).

3. K. Kadowaki, H. Yamaguchi, K. Kawamata, T. Yamamoto, H. Minami, I. Kakeya, U. Welp, L. Ozyuzer, A. Koshelev, C. Kurter, K.E. Gray, and W.-K. Kwok, Physica C468, 634 (2008).

4. K.E. Gray, A.E. Koshelev, C. Kurter, K. Kadowaki, T. Yamamoto, H. Minami, H. Yamaguchi, M. Tachiki, W.-K. Kwok, and U. Welp, IEEE Trans. Appl. Supercond. 19, 886 (2009).

5. Shizeng Lin, Xiao Hu, and Masashi Tachiki, Phys. Rev. B77, 014507 (2008).

6. Cihan Kurter, Kenneth E. Gray, John Zasadzinski, Lutfi Ozyuzer, Alexei E. Koshelev, Qing'an Li, T. Yamamoto, K. Kadowaki, Wai-Kwong Kwok, M. Tachiki, and Ulrich Welp, IEEE Trans. Appl. Supercond. 19, 428 (2009).

7. S. Guénon, M. Grünzweig, B. Gross, J. Yuan, Z.G. Jiang, Y.Y. Zhong, M.Y. Li, A. Iishi, P.H. Wu, T. Hatano, R.G. Mints, E. Goldobin, D. Koelle, H.B. Wang, and R. Kleiner, Phys. Rev. B82, 214506 (2010).

8. B. Wang, S. Guénon, B. Gross, J. Yuan, Z.G. Jiang, Y.Y. Zhong, M. Grunzweig, A. Iishi, P.H. Wu, T. Hatano, D. Koelle, and R. Kleiner, Phys. Rev. Lett. 105, 057002 (2010).

9. A. Grib, J. Scherbel, and P. Seidel, Phys. Status Solidi A198, 142 (2003).

10. A.N. Grib, P. Seidel, and J. Scherbel, Phys. Rev. B65, 094508 (2002).

11. A. Grib and P. Seidel, Phys. Status Solidi RRL3, 302 (2009).

12. K.K. Likharev, Dynamics of Josephson Junctions and Circuits, Gordon and Breach, Philadelphia (1991).

13. V.N. Gubankov, K.K. Likharev, and N.M. Margolin, Sov. J. Fiz. Tverd. Tela 14, 953 (1972) (in Russian).

14. U. Gottwick, R. Held, G. Sparn, F. Steglich, H. Rietschel, D. Ewert, B. Renker, W. Bauhofer, S. von Molnar, M. Wilhelm, and H.E. Hoenig, Europhys. Lett. 4, 1183 (1987). 